\title{
Design and Analysis Of Underwater Imaging Application susing High Frequency Array
}

\author{
Premsagar Konapally ${ }^{a}$, Dr. Mukesh Tiwari ${ }^{\text {b }}$ \\ a Research Scholar, Dept. of Electronics and Communication Engineering, \\ Sri Satya Sai University of Technology \& Medical Sciences, Sehore, Bhopal Indore Road, Madhya Pradesh, India \\ ${ }^{\mathbf{b}}$ Research Guide, Dept. of Electronics and Communication Engineering, \\ Sri Satya Sai University of Technology \& Medical Sciences, Sehore, Bhopal Indore Road, Madhya Pradesh, India
}

Article History: Received: 11 January 2021; Accepted: 27 February 2021; Published online: 5 April 2021

\begin{abstract}
Underwater picture preparing has a few applications in the field of maritime exploration work and logical applications, for example, archaeology, geography, underwater ecological appraisal, laying of significant distance gas pipelines and correspondence joins across the mainlands which request geo-referential looking over of the maritime bed and prospection of complex task. The lowering of a camera underwater requires satisfactory lodging utilizing high frequency. The moving of the camera with the assistance from distant spot or face to face at the site is similarly a perplexing undertaking. In any case, the significant test is forced by underwater medium properties. Underwater dimness picture upgrade has acquired far and wide significance with the quick advancement of present day imaging gear. Notwithstanding, the difference upgrade of single underwater dim picture is a difficult errand for logical investigation and computational applications. Versatile differentiation upgrade calculations to determine the picture fluffiness was proposed to suit underwater pictures with shifting difference. Fuzzy edge held intensification strategy had the option to furnish better upgrade with very much protected edge data and improved differentiation in examination with the fuzzy enhancement technique. Acoustic imaging systems are likewise valuable for reviewing or inspecting objects when water turbidity blocks the utilization of closed circuit television or other optical methods for survey. Likewise, light is weakened with a horizontal distance of proliferation, decreasing the light energy arriving at the camera, accordingly bringing about a deficiency of the normal tone.
\end{abstract}

Keywords: Underwater image processing, high frequency, computational applications, fuzzy edge.

\section{Introduction}

A high goal acoustic imaging system is a significant guide in turbid water where optical systems fizzle. The scope of appropriateness changes as indicated by the particular sensor received and the sign frequency portraying the sensor. The main specialized issues are the improvement of high thickness, minimal effort transducer clusters and interconnect; the related low force signal preparing hardware; picture handling calculations, and reduced and gap concealed acoustic focal point plans. As the ultrasound goes through water, it gets weakened corresponding to the frequency. Henceforth, there is a compromise among goal and infiltration profundity. The imaging system for the most part comprises of a projector, a recipient cluster, an acoustic focal point, Frontend gadgets and programming for signal molding and picture age. When planning a cluster for imaging, the elements to be considered incorporate the lower and upper frequency constraints, the greatest side projection level of the exhibit characterizing its dynamic reach, the cluster measurements, the estimation distance, the spatial goal and the size of the planning region. These amounts are exceptionally interconnected. The capacity and handiness of imaging calculations rely upon the quality (sensitivity, bandwidth, and dynamic scope) of the first reverberation signal. Underwater imaging sonar has wide applications including mine location, structure review, side sweep sonar and so on Underwater imaging sonar system comprise of high frequency sensors, beneficiary, transmitter, signal preparing and picture handling segments. Figure 2.1 shows the square outline of an imaging sonar system.

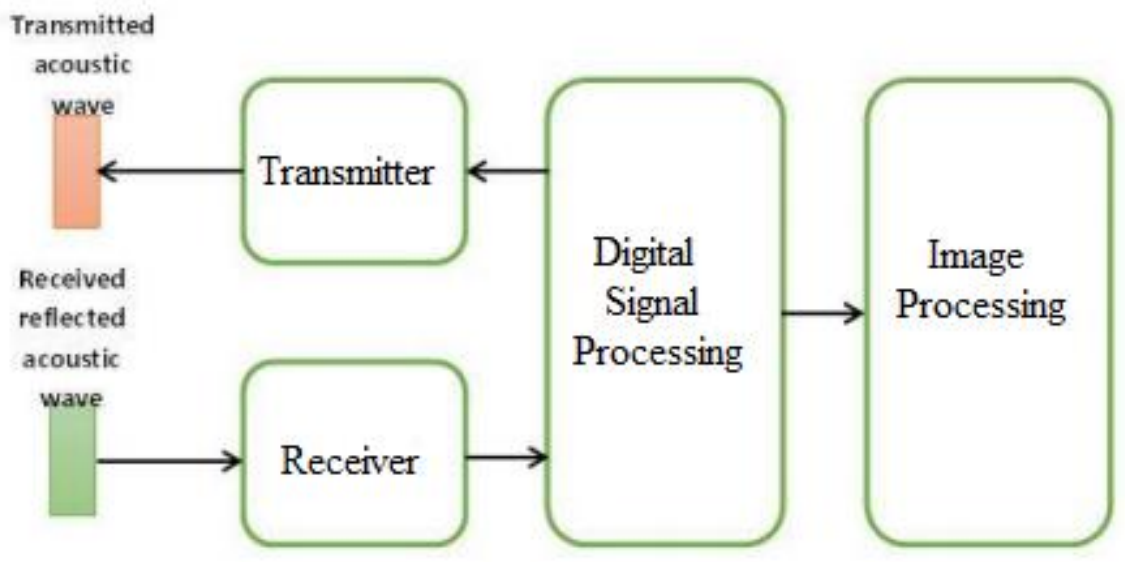

Figure 2.1. Block diagram of imaging sonar

Imaging sonar is a functioning sonar system, where a sign of high frequency is communicated over the medium and the reverberation is gathered by the beneficiary system. The transmitter comprises of a transmitter regulator and waveform age module and a force enhancer. Transmitter regulator and waveform age module is 
acknowledged utilizing a FPGA. The square chart of the pulser based transmitter area is appeared in Figure 2.2. Here the transmitter regulator and waveform age module creates the waveform with wanted frequency, beat width, Pulse Repetition Interval (PRI), transmission capacity and so on Created signal is intensified utilizing pulser chip to the necessary force level in order to energize sonar transducer. The working frequency of imaging sonar goes from many Kilo Hertz to Mega Hertz, giving high exactness in reach goal. So the transmitter area for a high frequency imaging sonar system should create high voltage excitation signs to drive ultrasonic transducers to defeat the reach restriction at high frequencies.

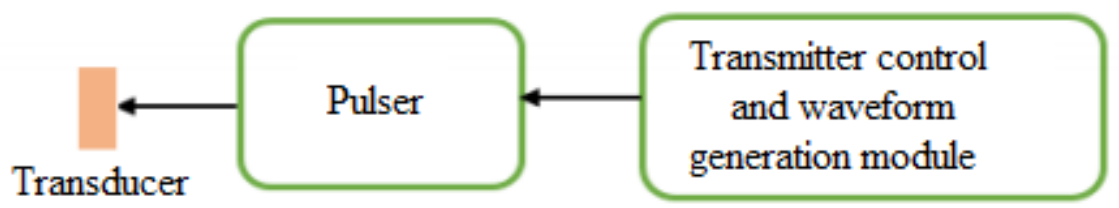

Figure 2.2. Block diagram of imaging sonar transmitter

Choice of sign waveforms decides the capacity of imaging sonar systems to determine focuses in reach and speed. Continuous Wave (CW) and Linear Frequency Modulated (LFM) wave are regularly utilized signs for underwater imaging sonar applications. Ultrasonic signs are normally utilized for imaging applications. Force prerequisite of clinical imaging systems are exceptionally less as their reach necessity is in couple of centimeters. The force prerequisite for underwater application is high when contrasted with medical imaging.

\section{Characteristics of underwater networks}

The characteristics of underwater networks are as follows

- Communication media:The correspondence in underwater climate includes electromagnetic, acoustic or radio waves. The acoustic method of correspondence is generally flexible and broadly utilized method of correspondence, as these signs are less influenced by the direct impedances in underwater climate and subsequently flags are less lessened.

- Noise: Noise is fundamentally ordered into human made commotion and surrounding clamor. The man made clamor is brought about by transportation action (structure fouling, cavitation), and apparatus (siphons, power plants). The surrounding commotion is identified with normal clamor made because of seismic, natural marvels, and hydrodynamics.

- Transmission loss:The transmission misfortune happens on account of two variables lessening and mathematical spreading. The lessening is incited because of transformation of acoustic energy into warmth and this increments with distance and frequency. The spreading of sound energy caused because of development of wavefronts alludes to mathematical spreading. The spread increments with distance of engendering and isn't subject to the frequency.

- Cost: The expense of underwater sensors is higher on the grounds that extra defensive sheaths must be given to sensors as these are inclined to disappointments as a result of consumption and fouling. The quantity of providers are likewise restricted.

- Multipath Propagation:Multipath engendering relies upon connect arrangement, this corrupts the acoustic sign in view of age of entomb image obstruction. Vertical and level channels are portrayed by scattering of time and multipath spreads individually.

\section{Difference between normal images and acoustic images}

The distance that sound goes in the sea changes extraordinarily, contingent fundamentally on water temperature and pressing factor. While compel keeps on expanding as sea profundity builds, the temperature of the sea just declines in a limited way, after which it remains moderately steady. These variables curiously affect how and how far sound waves travel. The contrasts between the ordinary picture and acoustic pictures are represented in Table 2.1

\begin{tabular}{|l|l|l|}
\hline Factors & Normal Images & Acoustic Images \\
\hline Source & Light & Sound \\
\hline Device & Digital Camera & Acoustic imaging system \\
\hline Image resolution & $\begin{array}{l}\text { Size of the image matrix, distance } \\
\text { between the object and camera, } \\
\text { shutter speed and aperture control }\end{array}$ & $\begin{array}{l}\text { Frequency, horizontal aperture, pulse } \\
\text { length, range scale, tow speed }\end{array}$ \\
\hline Scattering & $\begin{array}{l}\text { Distance between the object to the } \\
\text { lens }\end{array}$ & $\begin{array}{l}\text { Smooth surfaces }- \\
\text { reflection } \\
\text { Rough surfaces }- \text { specular reflection } \\
\text { and diffuse scattering }\end{array}$ \\
\hline
\end{tabular}




\begin{tabular}{|l|l|l|}
\hline Image formation & Sampling and Quantization & $\begin{array}{l}\text { Beamforming methods }- \text { Turns } \\
\text { recorded time series into image }\end{array}$ \\
\hline Shadows & $\begin{array}{l}\text { From lens hood, uncontrolled } \\
\text { lighting conditions }\end{array}$ & $\begin{array}{l}\text { The imaging geometry will cause } \\
\text { acoustic shadows from elevated objects } \\
\text { on the sea floor }\end{array}$ \\
\hline Distortion & $\begin{array}{l}\text { Due to noise, contrast, color, } \\
\text { exposure accuracy, lens flare etc. }\end{array}$ & $\begin{array}{l}\text { Due to an unstable towfish and } \\
\text { interference to acoustical noise }\end{array}$ \\
\hline \multirow{2}{*}{$\begin{array}{l}\text { Image quality } \\
\text { Depends upon the matrix size, } \\
\text { unsharpness, bit depth and noise in } \\
\text { the underlying image }\end{array}$} & $\begin{array}{l}\text { Depends upon the nature of underwater } \\
\text { objects, underwater noise, clutter } \\
\text { phenomena, geometries and timeliness }\end{array}$ \\
\hline Noise & $\begin{array}{l}\text { Gaussian, salt and pepper, shot, } \\
\text { uniform, film grain, periodic }\end{array}$ & $\begin{array}{l}\text { Ambient noise due to the following: } \\
\text { Hydrodynamic }- \text { tides, storm, rain } \\
\text { Biological }- \text { produced by marine } \\
\text { life } \\
\text { Man-made objects - ships }\end{array}$ \\
\hline
\end{tabular}

Table 2.1 Difference between normal images and acoustic images

\section{Underwater Imaging Challenges.}

Capturing underwater pictures is more troublesome than getting regular outside scene pictures. The main underwater picture was taken by W. Thompson in 1856 in England. There are numerous challenges for undersea optical imaging. The lowering of a camera underwater requires sufficient lodging. The moving of camera with the assistance from far off spot or face to face at the site is in like manner a mind boggling task. Be that as it may, the significant test is forced by underwater medium properties. The two principal underwater marvel influencing the result and visual perspective is light constriction and dissipating. As the distance among camera and item expands, the dissipated light delivers lower screen contrast in underwater pictures. As clear, dissipated light part doesn't convey any scene data and accordingly underwater optical imaging gets dreary. Exploration has been completed to measure the wideband weakening coefficients per shading divert in underwater pictures. In any case, these discoveries are generally restricted, as the boundaries become delicate to the first tone and the distance among item and camera. Dispersing is a significant issue which should be tended to while recuperating the dehazed picture from underwater pictures. As per the Jaffe-McGlamery underwater imaging model, the three segments, direct part, forward dispersing segment and back-dissipating segment comprise the complete brilliance of a picture that movements towards the camera. The presence of natural and inorganic particles suspended in the volume of water, crossed by the field of perspective on the camera and the light source is the reason for the dissipating marvel. This dissipating marvel is seriously influenced if turbidity is high. The level of dispersing relies upon shape and size qualities of particles.

\section{LITERATURE REVIEW}

Weilin (Will) Hou (2020): In this paper the author clarifies about the straightforward underwater imaging model. It is ordinarily realized that underwater imaging is thwarted by both retention and dispersing by particles of different starting points. Nonetheless, proof additionally demonstrates that the disturbance in regular underwater conditions can cause serious picture quality debasement. A model is introduced to remember the impacts of both molecule and choppiness for underwater optical imaging through optical exchange capacities to help evaluate the restricting variables under various conditions. The model uses Kolmogorov-type file of refraction power spectra found in the sea, alongside field models, to show that optical choppiness can restrict imaging goal by influencing high spatial frequencies.

G. Bianco et al (2020): The author clarifies about the recuperating right or if nothing else practical shades of underwater scenes is an extremely testing issue for imaging strategies, since brightening conditions in a refractive and turbid medium as the ocean are truly changed. The need to address shades of underwater pictures or recordings is a significant assignment needed altogether picture based applications like 3D imaging, route, documentation, and so forth. Many imaging improvement strategies have been proposed in writing for these reasons. The upside of these techniques is that they don't need the information on the medium actual boundaries while some picture changes can be performed physically (as histogram extending) or naturally by calculations dependent on certain rules as proposed from computational shading steadiness strategies. Quite possibly the most famous model depends on dark world speculation, which expects that the normal of the caught picture ought to 
be dim. A fascinating use of this supposition that is acted in the Ruderman adversary shading space l $\alpha \beta$, utilized in a past work for tone adjustment of pictures caught under hued light sources, which permits to isolate the luminance segment of the scene from its chromatic parts. In this work, we present the primary proposition for shading revision of underwater pictures by utilizing l $\alpha \beta$ shading space. Specifically, the chromatic segments are changed moving their disseminations around the white point (white adjusting) and histogram cutoff and extending of the luminance segment is performed to improve picture contrast. The exploratory outcomes exhibit the adequacy of this technique under dark world supposition and assuming uniform light of the scene. Additionally, because of its low computational expense it is reasonable for continuous execution.

\section{PROPOSED METHODOLOGY}

The examination and use of the underwater assets both living and non-living of the Indian coast line prompts geo-specialized, geophysical and oceanography study. Every one of these examinations are doable just with the assistance of underwater acoustics or hydro acoustics based instruments on the grounds that the sound wave just can travel bigger distance without obvious constriction in sea. As light constricts in more limited distance inside water, sound is utilized to deliver signals and to change over that into two-dimensional pictures. These days acoustic instruments can without much of a stretch guide or picture the sea floor, look beneath the ocean bed and measure different physical oceanographic boundaries with the exceptionally serious level of precision and generally high settling ability. For quite a while, underwater sonars have given a type of acoustic imaging in specific calculations. Both area output and side-looking sonars produce essentially a similar type of acoustic picture by sending a light emission at a brushing point to the base. At the point when no item is available, the base back disperses the energy as a pretty much uniform foundation. At the point when an article is available and rests over the base, it blocks and mirrors a portion of the sound sooner than would the base. Subsequently, more energy is reflected to frame a splendid spot at the scope of the article. Then, no energy is reflected-by the base inside the shadow of the article. As the bar is examined across the field of view, the bar converges various pieces of the item. Henceforth; the shadow of the item shows up on the showcase screen just past the brilliant spot. The brilliant spot demonstrates the presence of something, the shadow shows its state.

\section{High frequency Array Geometry}

The motivation behind an imaging cluster is to test the acoustic field over some spatial degree. A clear model is a two dimensional acoustically delicate plate which creates a consistently differing estimation of the sound field. An identical cluster from a data hypothesis perspective is a planar two dimensional routinely examined filled exhibit, for example, that appeared in the upper left of figure 4.1. To decrease the quantity of hydrophones required, a tested exhibit might be spatially under inspected by dispersing the hydrophones at distances more prominent than a half frequency. To forestall associating anyway the enlightening sound should be kept to a field of view (FOV) with half point $\theta$ given by transgression $\theta=\lambda / 2 \mathrm{~d}$. where $\mathrm{d}$ is the entomb hydrophone distance. 


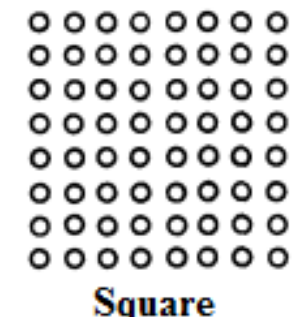

Square

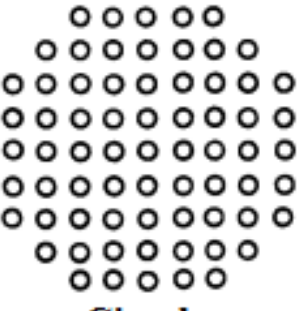

Circular

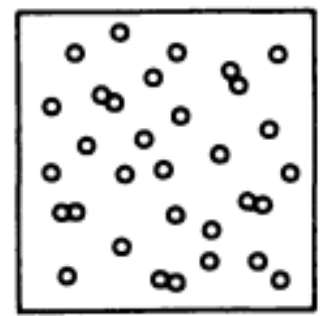

Random Thinned
Filled array vs Synthetic aperture array

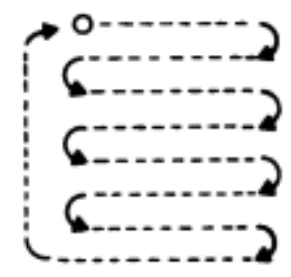

Raster

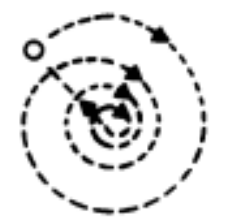

Spiral

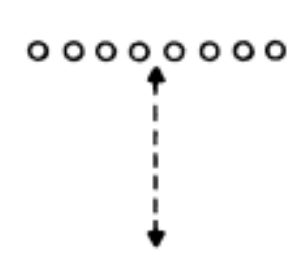

Linear

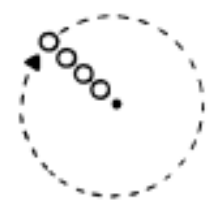

Rotational<smiles></smiles>

Cross

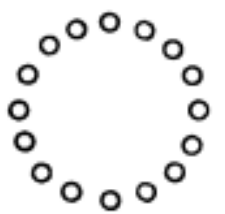

Annulus

\section{Semi Random Thinned}

Figure 4.1 A simple synthetic aperture patterns to synthesize the filled apertures shown, a random array and a partially random array

This is anything but a simple errand, as projector sidelobes falling external the FOV can reflect off items, hence creating got pictures on top free from the ideal pictures inside the FOV. Manufactured opening methods are additionally utilized in acoustic imaging. The expression "manufactured gap" signifies definitely more here than the restricted utilization it has accomplished as for engineered gap side output sonar. The side sweep manufactured opening procedure is indeed one exceptional instance of a more extensive idea that alludes to the reproduction of a filled exhibit by the shrewd utilization of some more modest cluster. Models incorporate a line exhibit checked opposite to its length, a point transducer filtered in a raster, and numerous different plans. In certain systems, the source is checked one way while the recipient filters toward another path. Whatever plan is utilized, the thought stays as before: supplant the huge exhibit of getting transducers with fewer sensors in some filtering course of action. The cost to be paid for the investment funds in transducers may incorporate expanded output time (which can prompt movement obscuring and more slow casing rate), or lower signal-to-clamor proportion. Modern sign handling procedures can make up for a portion of these exhibition costs, as long as the additional intricacy and cost are sensible.

The routinely dispersed clusters may likewise be supplanted by diminished, haphazardly divided, varieties of hydrophones. Interestingly with the consistently dispersed, polite side projection reactions of routinely divided clusters, arbitrary exhibits have sporadically separated sidelobes of unusual amplitudes. In any case, similarly as arbitrary clamor will display a reproducable RMS plentifulness, a total of sidelobes of an irregular cluster comply with comparable measurable laws in a polite way. The subsequent cluster can be appeared to display practically similar execution qualities as its consistently dispersed partner. For instance, goal is still conversely corresponding to the size of the opening. The semi-arbitrary cluster is gotten from the irregular exhibit simply by eliminating any hydrophones that exist in a half frequency of another with the end goal that they don't get autonomous data.

\section{Need for acoustic image preprocessing}

Acoustic picture preprocessing incorporates picture denoising and picture upgrade procedures. As per the working standards and working state of side sweep sonar, the wellspring of commotion is made out of the accompanying three sections. The first is the clamor from the climate. The sea climate is loud. There are commotions from wave, sound delivered by fish and other undersea animals, ocean water beating the stone and sound of boat cruising by. These commotions will be gathered by sonar. The second is the clamor from the hardware. The machine on the estimating transport, propeller, and the sonar fixed on the estimating transport and other hardware will make sound wave while working. These commotions will meddle sonar signals and make 
the side sweep sonar picture dirtied. The third is the clamor from the difference in temperature, saltiness and pressing factor of the ocean water. During the time spent transmission, these components will cause refraction, from which commotions come out.

Sonar picture of an article has less goal than the optical picture of a similar scene. In sonar pictures, the picture force is identified with spot commotion. Dot commotion in sonar pictures shows itself as multiplicative nonGaussian clamor in the power area and, subsequently, old style procedures of preparing and investigation may fall flat. In spite of the huge reach advantage over the standard vision, imaging sonar experiences a few downsides like lower Signal-to-Noise Ratio (SNR) because of acoustic wave obstruction called spot clamor and they contain practically homogeneous and finished areas with generally uncommon edges. The acoustic waves reflected from the ocean depths can have more prominent energy than the ones reflected from the impediments, prompting bogus deterrent location or to undetected ones. Different downsides incorporate the restriction of transducer size, bringing about a lower number of pixels and lower goal and the reach goal reliance. As the towfish is towed through the water, the horizontal sound pillars continuously filter an area of the seabed, while the recorder creates a line-by-line record of the backscattered signal.

The transducers are ordinarily formed and controlled to create a pillar, for each produced beat, which is thin the flat way and wide the vertical way. Because of the limited flat bar, returned energy is gotten from just a dainty portion of the ocean bottom. The wide vertical bar allows the ensonification of a huge fragment of the water section and permits the gathering of reflected energy from regions of the ocean bottom removed from the towfish. The spot impacts are more articulated in an extreme focus zone than in a low force zone. Dot commotion causes a discontinuity of items, and poor spatial directivity and goal, which brings about obscuring impacts around articles and impedes the understanding of the picture content. Since dot is hard to recognize from the genuine signs at the constraint of goal of the sonar, it demonstrates hard to eliminate without influencing fundamentally the picture. Preceding endeavoring division and highlight extraction in the picture, the spot commotion should be taken out. A harsher surface will backscatter more energy than a smooth surface. The strength of the sound recorded is changed over to shades of dark. An exceptionally solid return, say from uncovered stone is white. The direction of the objective comparative with the course of the approaching heartbeat will impact the force of the sign and subsequently the power of the picture.

\section{RESULTS}

Successful pre-preparing for underwater pictures is important for exact item acknowledgment. Here the aftereffects of different pre-handling channels in the wake of doing tests on underwater pictures are introduced. Two diverse filters were compared namely, homomorphic and bilateral filter to rectify the non-uniform illumination present in the underwater images. Light typically contrasts continuously across the picture when contrasted with reflectance segment which shifts strongly at object edges. This consistent variety in brightening was improved with the assistance of homomorphic channel, bringing about upgrade of foundation and edge data which is crucial for underwater pictures.

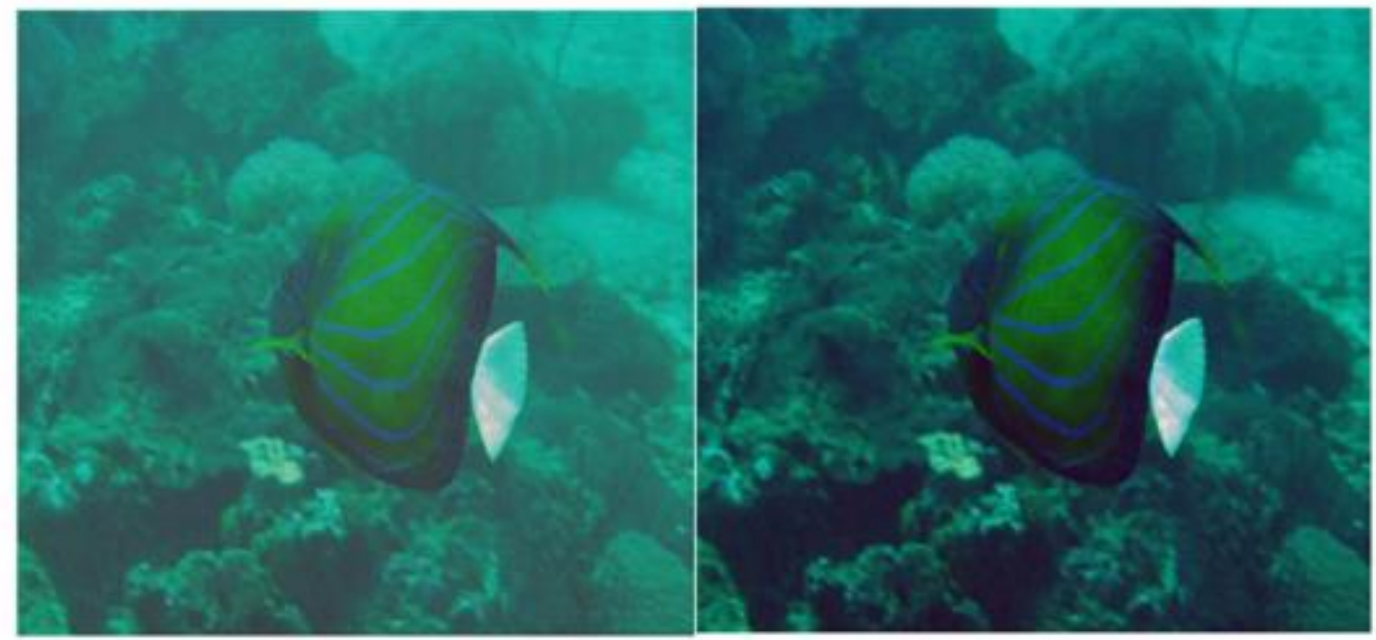

Figure 5.1 Non-uniform illumination correction. Left: Original image. Right: Homomorphic filtered image

Homomorphic channel is a honing channel and it will in general build the low frequencies in the picture and consequently in Figure 5.1 the foundation and fish show up outwardly more clear than the first picture. The dark foundation, the limit of the fish seem more obscure and even the blue shade of the fish scales seem upgraded. Different channels to be specific respective and anisotropic channel smoothens (blurs) the picture while holding solid edge data. Figure 5.2 shows the outcome subsequent to applying two-sided channel. Not at all like homomorphic channel, respective channel is a smoothing channel. Two-sided channel functions admirably for 
brightening revision in ordinary barometrical pictures. Since picture is as of now blurred, a smoothing channel like respective, may additionally expand the fogginess. However, the resultant picture didn't show up more obscured, as it played out a weighted normal procedure and is appeared in the correct hand side of Figure 5.2.

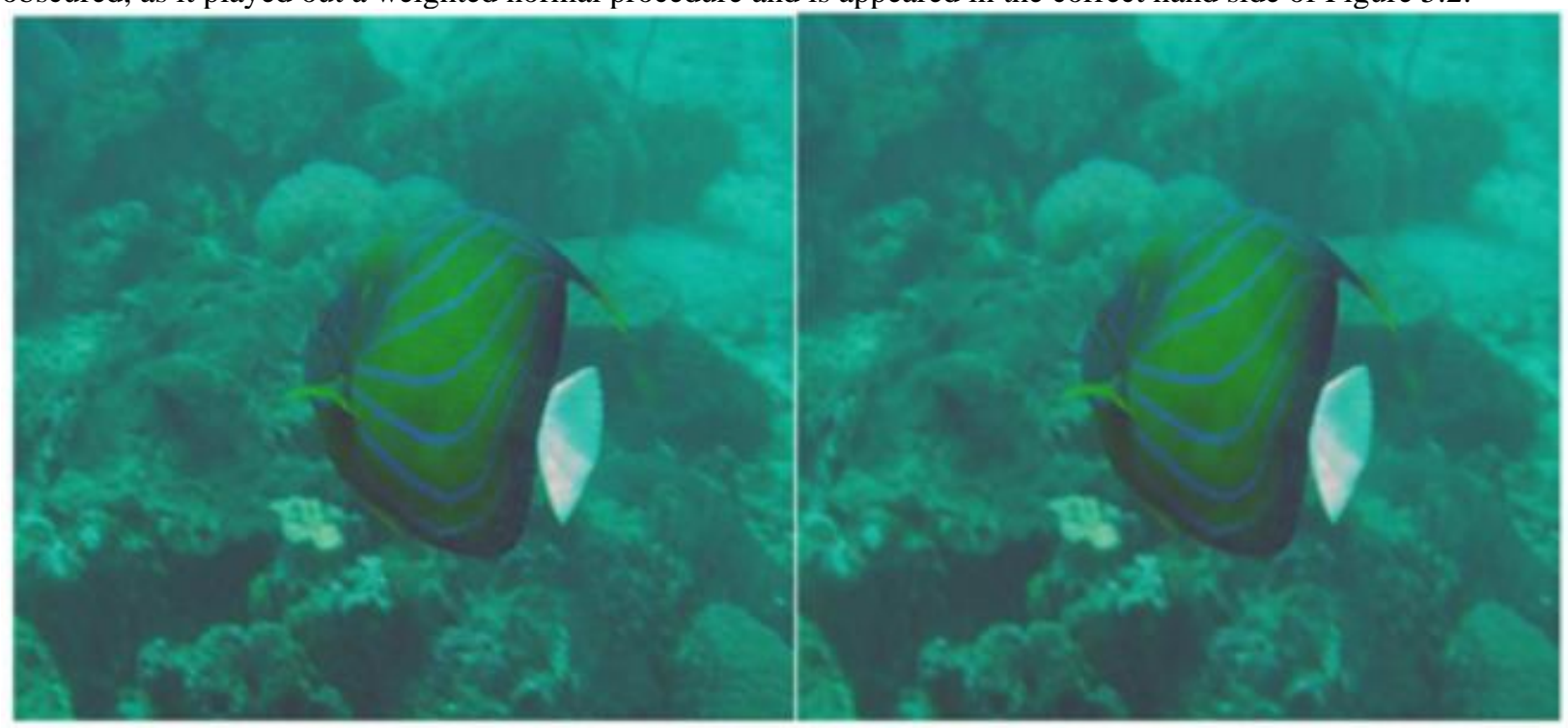

Figure 5.2 Non-uniform illumination correction. Left: Original image. Right: Bilateral filtered image

\section{CONCLUSION}

Underwater picture preparing is a possible apparatus for different military and regular citizen applications like examination of greenery, fisheries, coral reef research, seabed investigation, the quest for transport wrecks up to the investigation of polymetallic knobs. This paper has looked to introduce a concise outline of the mind boggling field of underwater acoustic imaging. There are numerous potential methods, advancements and methodologies for planning and building underwater acoustic imaging systems, and a couple of them have been attempted by different gatherings at different occasions. To address the non-uniform enlightenment present in the pictures, homomorphic and reciprocal channel were assessed. Moreover, to dispose of the commotion residuals, Haar and Symlet wavelet denoising were applied and assessed. To evaluate the degree of differentiation improvement, histograms were registered when upgrade. The picture pixels were additionally ordered into edge and clamor utilizing fluffy participation capacities. While smoothing, edge pixels were held and commotion pixels alone were smoothened..

\section{REFERENCES}

1. Hou, W. (Will). (2020). A simple underwater imaging model. Optics Letters, 34(17), 2688.

2. G. Biancoa, M. Muzzupappaa, F. Brunoa , R. Garciab , L. Neumann (2020), "A NEW COLOR CORRECTION METHOD FOR UNDERWATER IMAGING". The International Archives of the Photogrammetry, Remote Sensing and Spatial Information Sciences, Volume XL-5/W5

3. Schechner. Y., Karpel. N. (2018). “Clear Underwater Vision.” Computer Vision and Pattern Recognition, $1: 536-543$.

4. T.W. Cronin and N. Shashar. (2019). "The linearly polarized field inclear, tropical marine waters: spatial and temporal variation of light intensity, degree or polarization and e-vector angle."J. Experim. Biol. 204, 2461-2467

5. Motwani, Mukesh C., Mukesh C. Gadiya, Rakhi C. Motwani, and Frederick C. Harris. (2020). "Survey of image denoising techniques.” In Proceedings of GSPX, 27-30

6. S. Bazeille, I. Quidu, L. Jaulin. (2019). "Color-based underwater object recognition using water light attenuation," Intelligent Service Robotics, 5(2):109-118

7. Kesavan, S., Saravana Kumar, E., Kumar, A., \& Vengatesan, K. (2019). An investigation on adaptive HTTP media streaming Quality-of-Experience (QoE) and agility using cloud media servicesInternational Journal of Computers and Applications, 1-14.

8. C. Srisailam, P. Sharma, and S. Suhane. (2019). "Color Image Denoising Using Wavelet soft Thresholding," International Journal of Emerging Technology and Advanced Engineering, 4(7):475-478.

9. Ghada S. K., Ziad M. A. (2018). "Enhancement of Underwater Image using Fuzzy Histogram Equalization," International Journal of Applied Information Systems, 6(6).

10. X. Wu, H. Li. (2019). "A Simple and Comprehensive Model for Underwater Image Restoration", IEEE International Conference on Information and Automation, 699-704 
11. Kyung min Han, Hyun taek Choi. (2020). "Shape Context Based Object Recognition and Tracking in Structured Underwater Environment". IEEE International Geoscience and Remote Sensing Symposium (IGARSS). 617-620.

12. Kumar, A., Vengatesan, K., Rajesh, M., \& Singhal, A. (2019). Teaching literacy through animation \& multimediaInt. J. Innovative Technol. Exploring Eng, 8(5), 73-76 